\title{
a-Alkenoyl Ketene S,S- and N,S-Acetals As Starting For Unexpected and Novel Synthesis of $\mathrm{N}$-Heterocycles
}

\author{
Ahmed M. M. El-Saghier ${ }^{a *}$, Mansour A. Makhlouf ${ }^{\mathrm{b}}$, NaglaaF.H.Mahmoud ${ }^{\mathrm{c}}$ and Wedad A. El- \\ Adawish $^{\text {d }}$ \\ ${ }^{a)}$ Department of Chemistry, Faculty of Science, Sohag University, Sohag, Egypt, \\ el_saghier@yahoo.com \\ b) Department of Chemistry, Faculty of Science, Tripoli University, Tripoli, Libya \\ ${ }^{c}$ Department of Chemistry, Faculty of Science, Ain Shams University, Cairo, Egypt \\ d)Department of Chemistry, Faculty of Science, Al-Zawia University, Al-Zawia, Libya
}

\section{ABSTRACTr}

A series of $N$-heterocycles such as diazaspiro-[4.5]decane, pyrazolo[4,3-d][1,2]-diazepine, imidazo[3,2,1ij][1,8]naphthyridine derivatives or pyrazolo[3,4-b]pyridin-4-ol were synthesized using $\alpha$-alkenoyl-, $\alpha, \alpha$-dialkenoyl ketene-(S,S)acetals 2a-d, 3a-c or $\alpha$-dialkenoyl ketene- $(N, S)$-acetal 12 as starting materials. The biological activity of some new synthesized compounds have been investigated.

\section{Indexing terms/Keywords}

Oxo-ketene Acetals; $\alpha$-Alkenoyl ketene -S,S-acetal; $\alpha$-Alkenoyl keten-N,S-acetal; Diazaspiro[4.5]decane Pyrazolodiazepine; Pyrazolopyridine

\section{Academic Discipline And Sub-Disciplines}

Provide examples of relevant academic disciplines for this journal: E.gScience, Chemistry

\section{SUBJECT CLASSIFICATION}

E.g., Hetrocyclic Chemistry

\section{TYPE (METHOD/APPROACH)}

Experimental;

\section{Council for Innovative Research}

Peer Review Research Publishing System

Journal: Journal of Advances in Chemistry

Vol. 6, No. 1

editor@cirworld.com

www.cirworld.com, member.cirworld.com 


\section{INTRODUCTION}

Indeed, the first synthesis of an $\alpha$-oxo ketene-(S,S)-acetal was reported in 1910 by Kelber and co-workers [1]. This compound was prepared by alkylation of $\beta$-oxodithioic acid which was obtained in poor yield by reaction of an aryl ketone with $\mathrm{CS}_{2}$ and $\mathrm{KOH}$ at $100{ }^{\circ} \mathrm{C}$ followed by neutralization with sulfuric acid. In the early 1960s Thuillier and Vialle successfully prepared a-oxo ketene-(S,S)-acetals directly from ketones in good yields by using sodium tert-amylate as the base and two equivalents of an alkyl halide [2,3]. Subsequent workers devoted their efforts towards improving the synthetic methodology and achieved mild reaction conditions, high yields and wide generality to wide substrates for the synthesis of $\alpha$-oxo ketene- $(S, S)$-acetals [3]. However, there are only a few reports so far on the synthesis of $\alpha$-oxo ketene$(S, S)$-acetals in aqueous media, [4] and none in water.

The use of water as a solvent in organic chemistry was rediscovered in the 1980s in Breslow's work, which showed that hydrophobic effect could strongly enhance the rates of some organic reactions [5]. Organic reactions carried out in water, without the use of any organic solvent, can also be beneficial because water is an easily available, cheap, safe and environmentally benign solvent [6]. A clean, facile and practical synthesis of a-oxo ketene-(S,S)-acetals based on the reaction of $\alpha$-dicarbonyl compounds with carbon disulfide and alkyl bromide catalyzed by tetrabutyl ammonium bromide (TBAB) in the presence of potassium carbonate in water $[7,8]$.

El-Saghier et.al, [9] reported that the synthesis 2-acetyl-2-oxopropylidene-(S,S)-acetal was obtained via reaction of acetyl acetone, $\mathrm{CS}_{2}$ and two moles of methyl iodide in one-pot reaction using phase transfer Conditions PTC $\left[\mathrm{K}_{2} \mathrm{CO}_{3} /\right.$ benzene/tetrabutyl ammonium bromide (TBAB)] in almost $100 \%$ yield.

Liu et. al.[10] , they reported the important synthesis of novel $\alpha$-alkenoyl ketene-(S,S)-acetals in aqueous media as intermediate in organic synthesis. They investigated the aldol condensation reactions of $\alpha$, $\alpha$-diacetyl ketene-( $S, S)$-acetals with various selected aryl aldehydes affording a variety of novel $\alpha$-alkenoyl ketene-(S,S)-acetals in water.

The important synthetic utility of such intermediate [11-17] showed that a-alkenoyl ketene-(S,S)-acetals containing a dienone moiety showed promising structural features as novel intermediates for: (1) double Michael acceptors serving as five carbon 1,5-bielectrophilic species, (2) dense and flexible substitution patterns and (3) good leaving alkyl thio groups that can be subjected to a nucleophilic vinyl substitution (SNV) reaction. Consequently, they developed a novel synthetic strategy for the construction of highly substituted six-membered carbocycles and heterocycles, relying upon the utilization of $\alpha$-alkenoyl ketene-S,S-acetals as a five carbon 1,5-bielectrophilic species informal [5+1] annulation with various carbon, nitrogen and sulphur nucleophiles, respectively [18] .

\section{Results and Discussions}

The synthesis and application of $\square$-oxo ketene-(S,S)-acetals as starting material in heterocyclic synthesis have been reported in the recent work in our laboratory [9,22-29] and elsewhere.[1-4, 20,21] Following the procedure described by Liu et. al [10], $\alpha$-alkenoyl- and $\alpha, \alpha \square$-dialkenoyl ketene-(S,S)-acetals 2, 3 were easily prepared via direct aldol condensation reactions of some $\alpha, \alpha^{\prime}$-diacetyl ketene- $(S, S)$-acetals 1 with aromatic aldehydes.

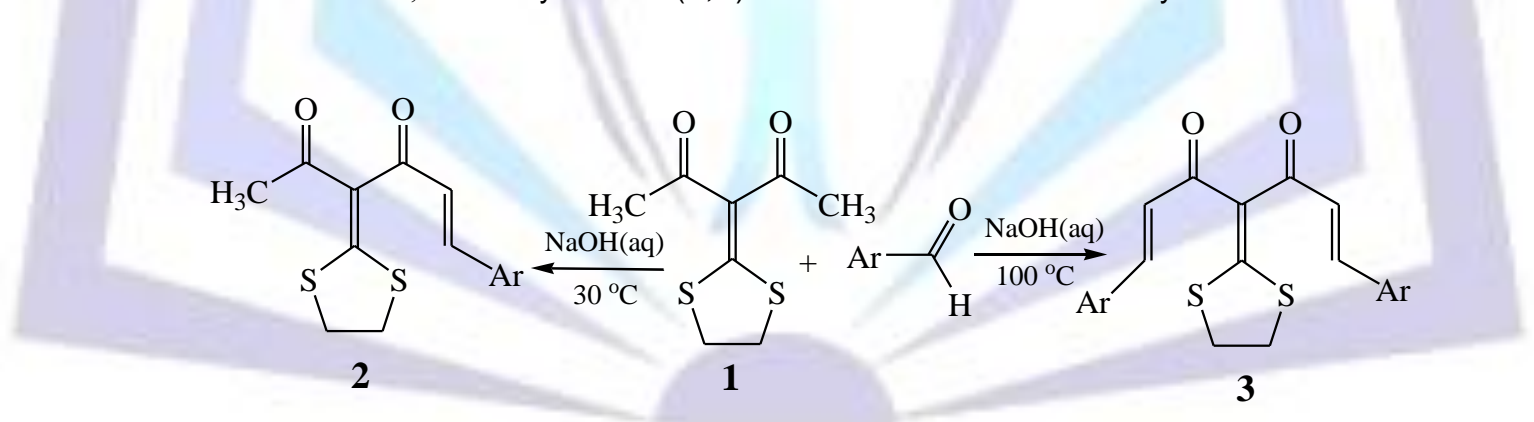

In the present work, we found that the use of $\alpha$-alkenoyl ketene-( $S, S)$-acetals 2 or $\alpha, \alpha^{\prime}$-dialkenoyl ketene- $(S, S)$-acetals $\mathbf{3}$ as intermediate in heterocylic synthesis afforded an expected and unexpected novel $N$ hetercycles.

A solution of 2-(propan-2-ylidene)-1,3-dithiolane 2a-d were allowed to react with 2-aminothiophenol in DMF in one portion at room temperature afforded (Z)-6-(2-aminophenythio)-3-(1,3-dithiolan--2-ylidene)-6-arylhex5-ene-2,4-dione 5a-d instead of the expected 3-acetyl-5,6-dihydro-6-arylbenzthiazolo[3,2-a]-pyridine-4-one 4a-d. 


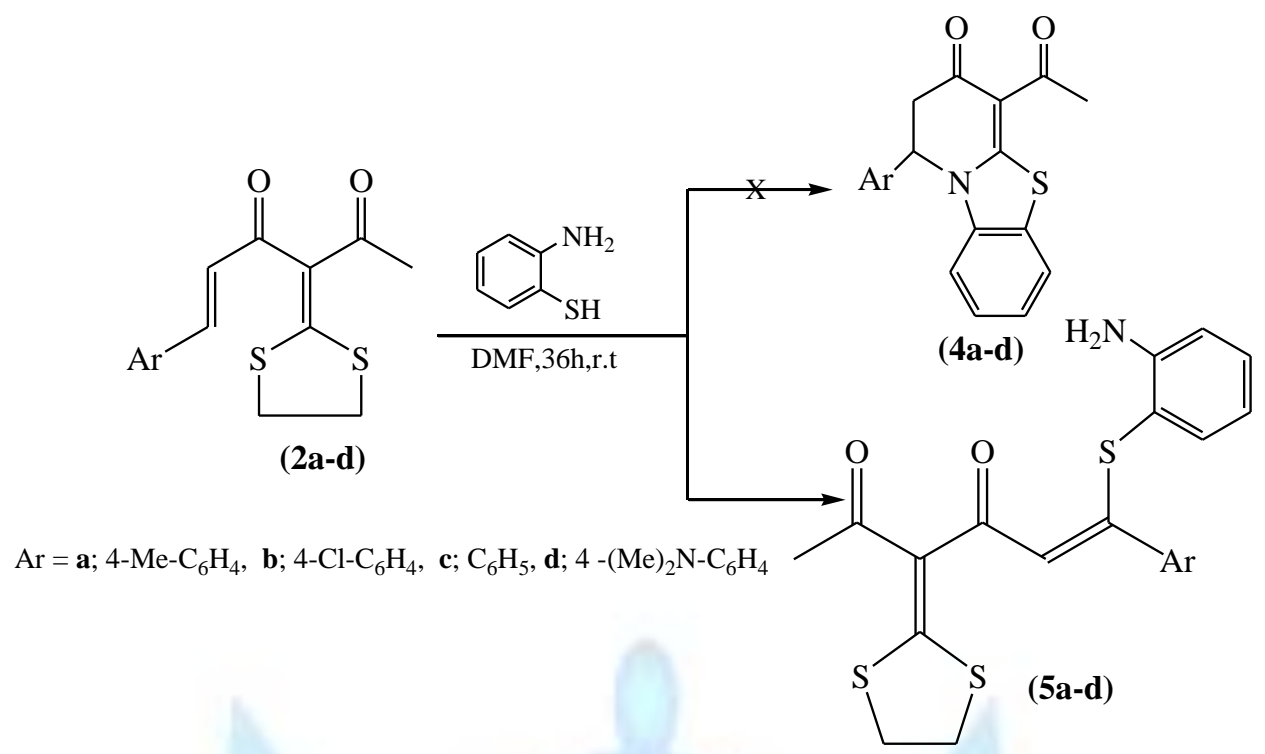

The spectral data of the synthesized compounds are in accordance with the proposed structures. The $I R\left(v \mathrm{~cm}^{-1}\right)$ spectra of compounds 4a-d showed new bands at $3370-3280 \mathrm{~cm}^{-1}$ for $\mathrm{NH}_{2}$ groups; and the ${ }^{1} \mathrm{H}-\mathrm{NMR}$ spectra showed a new singlet at 4.60-4.40 ppm for $\left(\mathrm{NH}_{2}\right)$.

Unexpected synthesis of 1,4-diazaspiro[4.5]decane-6-carbonirile derivatives 6a-d were obtained via reaction of 3-(1,3dithiolan-2-ylidene)-6-aryl-hex-5-ene-2,4-dione 2a-d with ammonium acetate in DMSO under heating at $100{ }^{\circ} \mathrm{C}$ instead of 8-acetyl-2,3,5,6-tetrahydro-5-arylimidazo-[1,2-a]pyridin-7(1H)-one 7a-d.

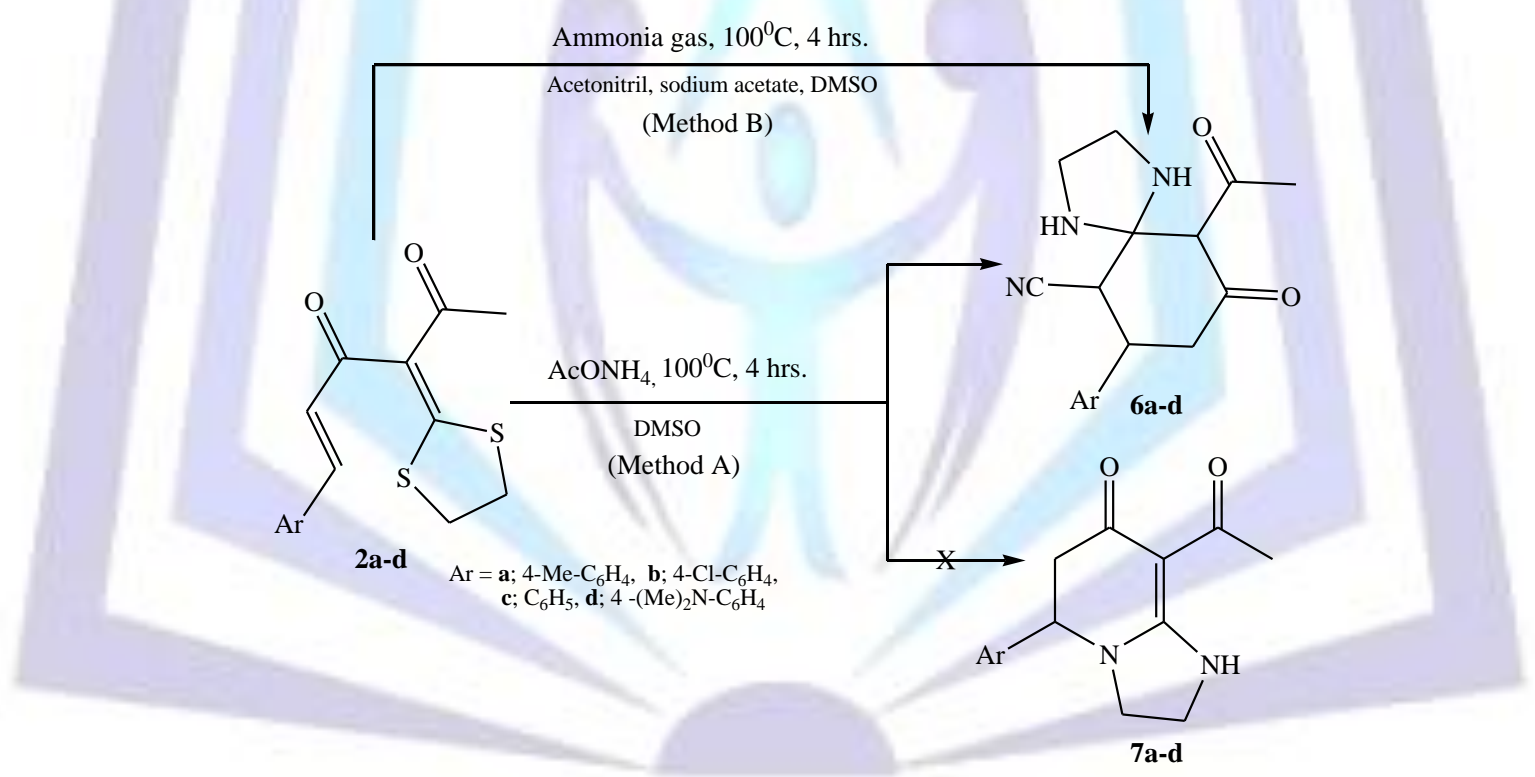

The reaction mechanism was assumed to proceed via a nucleophilic attack of ammonia (which produced from decomposition of $\mathrm{AcONH}_{4}$ ) at the ethylenic bond of ketene-(S,S)-acetals followed by elimination of $\mathrm{H}_{2} \mathrm{~S}$ to give the corresponding ketene- $(N, N)$-acetals. Under the higher temperature, the ammonium acetate is converted into acetonitrile by lost of two moles of water (Figure 1). Under the reaction condition, the carbanion of acetonitrile which was generated under basic condition attack at the ethylenic bond of ketene- $(N, N)$-acetals followed by another nucleophilic attack of the newly formed carbanion at the ethylenic bond of $\alpha, \beta$-unsaturated alkenoyl and cyclization. 


\begin{tabular}{|c|c|c|c|c|}
\hline \multirow{2}{*}{$\mathrm{CH}_{3} \mathrm{COONH}_{4}$} & heat & \multirow{2}{*}{$\mathrm{CH}_{3} \mathrm{CONH}_{2}$} & heat & \multirow{2}{*}{$\mathrm{CH}_{3} \mathrm{CN}$} \\
\hline & $-\mathrm{H}_{2} \mathrm{O}$ & & $-\mathrm{H}_{2} \mathrm{O}$ & \\
\hline
\end{tabular}<smiles>NCC(=O)C(=C1SCCS1)C(C(N)=O)C(=O)/C=C/[Al]</smiles>

2a-d<smiles>CC(=O)C(C(C)=O)C1(CC#N)NCCN1</smiles>

Figure 1<smiles>CCCCC(=O)C(C(C)=O)C1(CCC)NCCN1</smiles><smiles>CC(=O)C(C(C)=O)=C1NCCN1</smiles><smiles>CCCC1(C(C(C)=O)C(=O)/C=C/[Al])NCCN1</smiles><smiles>C[Al]C1CC(=O)C(C(C)=O)C(C#N)C12NCCN2</smiles>

6a-d

Table1, Comparison synthesis of compounds 6a-d (method A and B)

\begin{tabular}{|c|c|c|c|}
\hline Entry & Comp. No & Conditions & Yield \% \\
\hline 1 & $6 a$ & $\mathrm{AcONH}_{4} / \mathrm{DMSO} / 4 \mathrm{hrs} . / 100^{\circ} \mathrm{C}$ & 75 \\
\hline 2 & $6 b$ & $\mathrm{AcONH}_{4} / \mathrm{DMSO} / 4 \mathrm{hrs} . / 100^{\circ} \mathrm{C}$ & 84 \\
\hline 3 & $6 c$ & $\mathrm{AcONH}_{4} / \mathrm{DMSO} / 4 \mathrm{hrs} . / 100{ }^{\circ} \mathrm{C}$ & 58 \\
\hline 4 & $6 d$ & $\mathrm{AcONH}_{4} / \mathrm{DMSO} / 4 \mathrm{hrs} . / 100^{\circ} \mathrm{C}$ & 63 \\
\hline 5 & $6 a$ & $\mathrm{NH}_{3(\mathrm{~g})}, \mathrm{CH}_{3} \mathrm{CN}, \mathrm{AcONa}$ & 69 \\
\hline 6 & $6 b$ & $\mathrm{NH}_{3(\mathrm{~g})}, \mathrm{CH}_{3} \mathrm{CN}, \mathrm{AcONa}$ & 77 \\
\hline 7 & $6 c$ & $\mathrm{NH}_{3(\mathrm{~g})}, \mathrm{CH}_{3} \mathrm{CN}, \mathrm{AcONa}^{\star}$ & 55 \\
\hline 8 & $6 d$ & $\mathrm{NH}_{3(\mathrm{~g})}, \mathrm{CH}_{3} \mathrm{CN}, \mathrm{AcONa}$ & 59 \\
\hline
\end{tabular}

Method (B)

This mechanism was proved by the synthesis of compounds $\mathbf{2 a - d}$ via reaction of the starting materials $2 \mathbf{a}-\mathbf{d}$ with acetonitrile and ammonia gas at $100^{\circ} \mathrm{C}$ in DMSO for about $4 \mathrm{hrs}$. in the presence of sodium acetate as a base (method B entry $5-8)$. The yield of the reaction is moderately high in an entry (1-4) than in an entry (5-8) where compound $6 \mathbf{b}$ was 84 $\%$ in entry 3 but was $77 \%$ in entry 6 (Table 1 ).

Also, the unexpected (3aZ,5Z,7Z)-3-methylpyrazolo[4,3-d][1,2]-diazepine 8 was prepared via reaction of $(E)$-3-(1,3dithiolan-2-ylidene)-6-(4-(N,N-dimethylamino)phenylhex-5-ene-2,4-dione $2 \mathbf{d}$ with hydrazine hydrate was carried out in DMSO at $120^{\circ} \mathrm{C}$ in an oil bath with stirring for about $4 \mathrm{hrs}$.

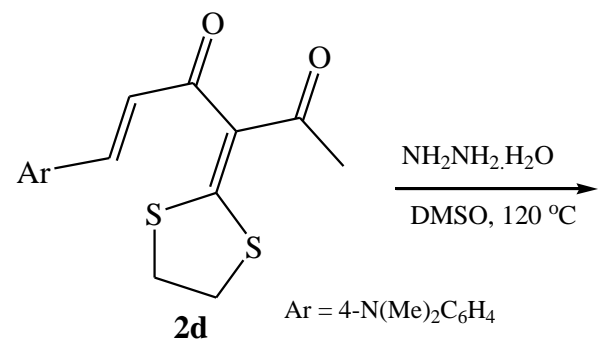<smiles>Cc1nnc2ccnncc1-2</smiles> 
The ${ }^{1} \mathrm{H}-\mathrm{NMR}$ spectra showed the disappearance of the aromatic and $\mathrm{CH}_{2}$ signals and appearance of ethylic $\mathrm{CH}$ of diazepines ring. The ${ }^{13} \mathrm{C}-\mathrm{NMR}$ spectra showed the disappearance carbonyl group signals and the appearance the signals correspondence to the compound 8 .

The reaction mechanism is assumed to proceed via a nucleophilic attack of two moles of hydrazine, the first attack at the ethylenic double bond of ketene-(S,S)-acetals followed by cyclization to diazepine ring with elimination of aromatic ring, and the second mole attack at carbonyl carbon with elimination of two moles of moles of water followed by elimination of dithioethanol to afford the desired compound 8 (c.f. Figure 2).<smiles>CC(=O)C(C(C)=O)=C1SCCS1</smiles>

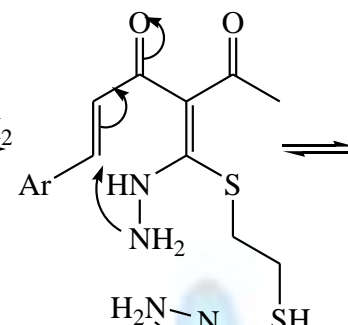<smiles>CC(=O)C1=C(SCCS)NNC(C)(C)C=C1O</smiles><smiles>C=CC(C)=C1C(=O)C=CNNC1SCCNN</smiles><smiles>C=CSC1=C2C(C)=NNC2(C)C=CNN1</smiles><smiles>C=CSC1=NNC=Cc2[nH]nc(C)c21</smiles><smiles>C/C(=N/N)C1=C(SCCS)NNC=CC1(C)C(=O)O</smiles><smiles>C#CC1=NN=C2C=CNNC(SCCS)=C12</smiles><smiles></smiles>

Figure 2

On the other hand, compound $2 \mathrm{a}$ reacted with phenyl hydrazine at $120{ }^{\circ} \mathrm{C}$ in DMSO to afford the corresponding the imidazodiazepinone 9 .

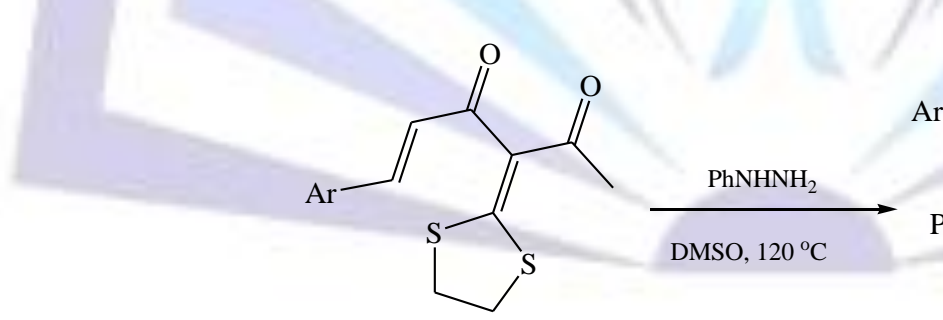

$\mathbf{2 a}$

$$
\mathrm{Ar}=p-\mathrm{Me}-\mathrm{C}_{6} \mathrm{H}_{4}
$$

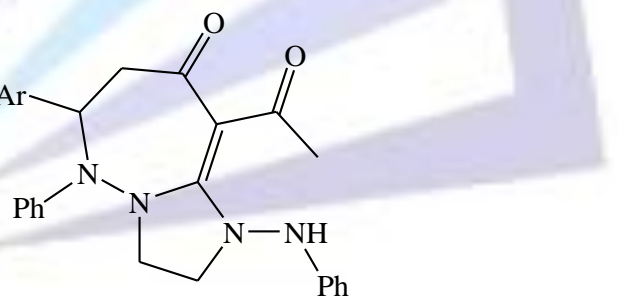

9

The IR spectrum data for compound 9 showed a new band at $3194 \mathrm{~cm}-1$ for $\mathrm{NH}$ group. The ${ }^{1} \mathrm{H}-\mathrm{NMR}$ showed a signal at $7.70 \mathrm{ppm}$ as a singlet for $1 \mathrm{H}(\mathrm{NH})$; and $7.60-6.80 \mathrm{ppm}$ as a multiplet for aromatic protons; $3.40 \mathrm{ppm}$ for $\left(\mathrm{CH}_{2}-\mathrm{CO}\right)$. The ${ }^{13} \mathrm{C}$-NMR) spectrum showed the appeared in opposite direction when applying DEPT technique; a new signal at 31.12 ppm for C7 of diazepin ring..

On the other hand, way, $\alpha, \alpha^{\prime}$-dialkenoyl ketene-(S,S)-acetals 3a-c were allowed to react with ethylenediamine in one pot reaction at room temperature, afforded the corresponding 1,2,4,9-tetrahydro-4,9-diarylylimidazo[3,2,1ij][1,8]naphthyrid-ine-6,7-diol derivatives 10a-c. 

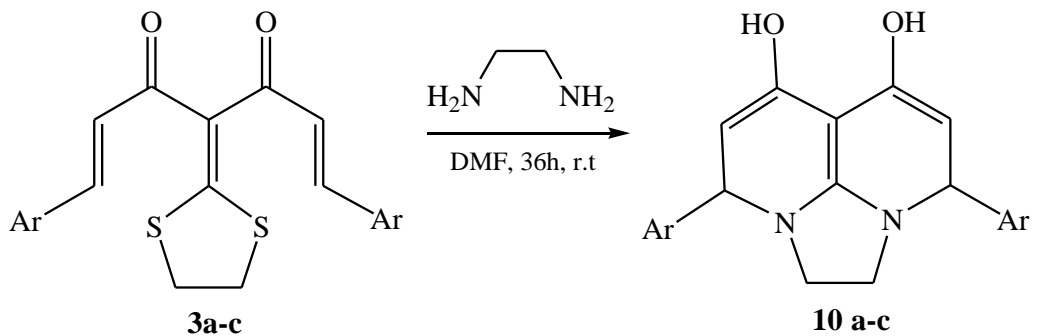

Ar, $\mathrm{a} ;=4-\mathrm{MeC}_{6} \mathrm{H}_{4}, \mathrm{~b}=4-\mathrm{ClC}_{6} \mathrm{H}_{4}, \mathrm{c}=\mathrm{C}_{6} \mathrm{H}_{5}$

The spectral data of the synthesized compounds 10a-c are in accordance with the proposed structures. The IR v cm${ }^{-1}$ spectra for 10a-c showed new bands for $\mathrm{OH}$ group at $3286-3282 \mathrm{~cm}^{-1}$ and the ${ }^{1} \mathrm{H}-\mathrm{NMR}$ spectra showed a new signals at 9.60-9.50 ppm as singlet for $\mathrm{OH}$ group; a doublet at 3.80-3.70 ppm for $(2 \mathrm{H}, 2 \mathrm{CH}-\mathrm{Ar})$ and appeared as revered to opposite direction when applying DEPT technique; a singlet at $2.01 \mathrm{ppm}$ for $\left(2 \mathrm{CH}_{3}\right)$ of compound 10a.

Also, 3a-c were allowed to react with 2 moles of 2-aminothiophenol under the same condition and reaction mechanism of compound 5 to afford (1Z,6Z)-1,7-bis(2-aminophenylthio)-4-(1,3-dithiolan-2-ylidene)-1,7-diarylhepta-1,6-diene-3,5-dione 11a-c

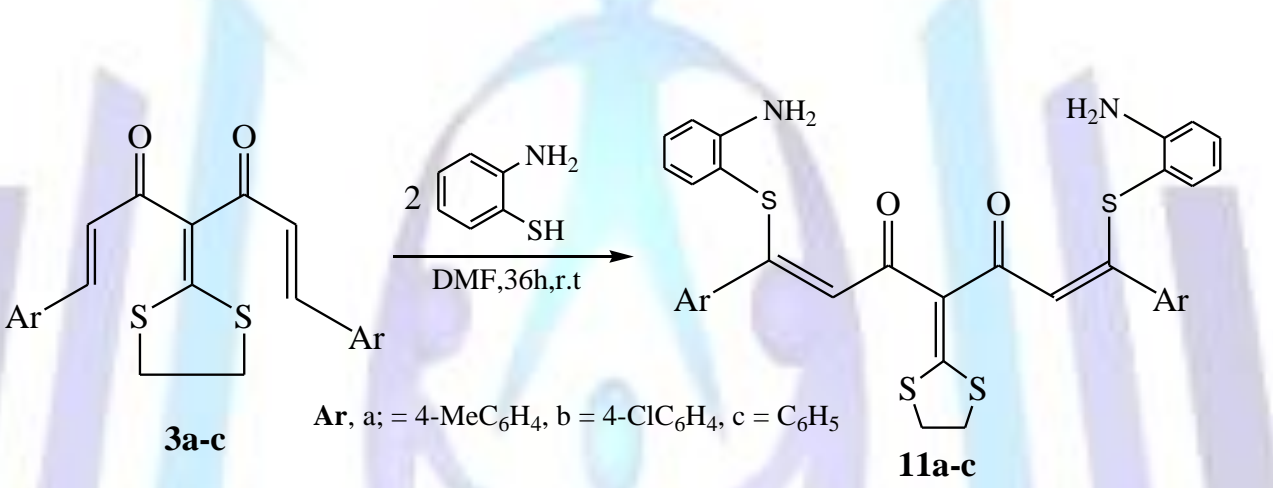

In the same strategy, reaction of $\square$-dialkenoyl ketene-(N,S)-acetal 12 with hydrazine hydrate was heated at $120{ }^{\circ} \mathrm{C}$ in DMSO for 2 hrs gave 3-methyl-6-p-tolyl-6H-pyrazolo[3,4-b]pyridin-4-ol 13

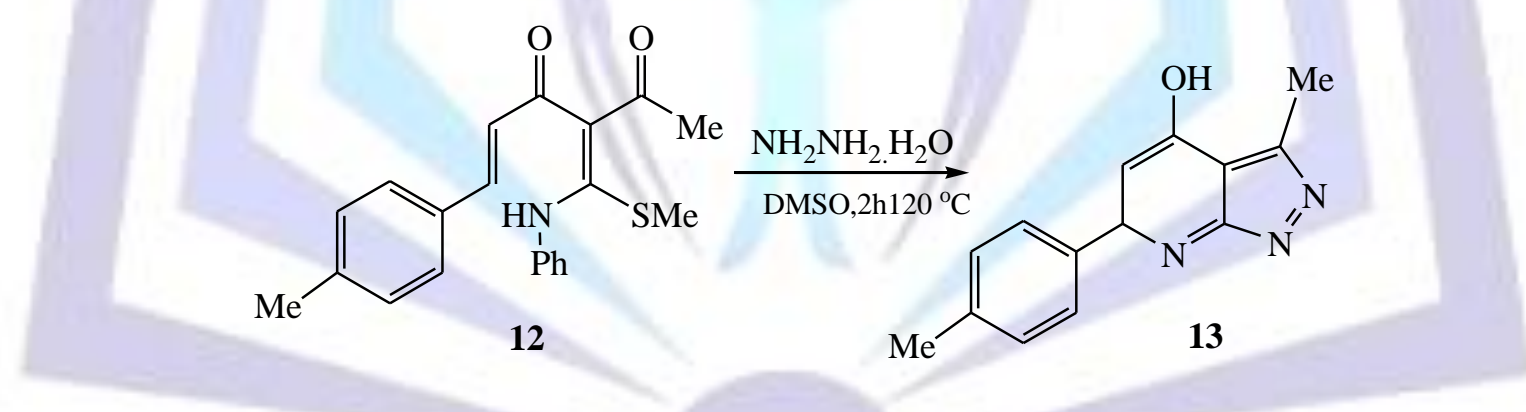

The spectrum date of compound $\mathbf{1 3}$ is accordance with the proposed structures.

The suggested reaction mechanism may include two steps. The first involves nucleophilic attack of amino group of hydrazine at the ethylenic bond with elimination of $\mathrm{MeSH}$ followed by nucleophilic attack of the other amino group at the carbonyl group followed by cyclization to pyrazole ring. The second step involved a nucleophilic attack of the amino group of a second mole of hydrazine at $\square \square$-unsaturated bond followed by a nucleophilic attack of the amino group at the ethyleinc bond with elimination of aniline followed by elimination of $\mathrm{NH}_{3}$.

\section{Biological Activity Assessment.}

The biological activity of the compounds (5b-d, $\mathbf{1 1 b}, \mathbf{c}$ and $\mathbf{1 0 b}, \mathbf{c})$ given in Tables $(\mathbf{2}$, and $\mathbf{3})$ regard less their relatedness and nuclei were assessed using the paper disc assay procedure adapted by Baur et al.[28] (1966). This method was minorly modified and adapted to cover all compounds under investigation.

Compounds were dissolved in the suitable organic solvents to make stock solutions of final concentrations of 1000 and down to $100 \mu \mathrm{g} / 20 \mu \mathrm{L}$. Paper disks of $8 \mathrm{~mm}$ diameter (Whatmann No. 1) are loaded with $20 \mathrm{ml}$ of each individual compound under testing, solvent was evaporated under a stream of hot air.

Using sterile forceps discs were aseptically applied onto the surface of nutrient-agar plates (12 cm/diameter) previously inoculated with $0.25 \mathrm{ml}$ of $24 \mathrm{hrs}$ culture of the test organism (two Gram-positive or two Gram-negative species). Plates 
were then left for $1 \mathrm{hr}$ at $4{ }^{\circ} \mathrm{C}$ for better diffusion of the loaded test compounds, and then transferred to an incubator to grow for $24 \mathrm{hrs}$ at $37^{\circ} \mathrm{C}$. At the end of incubation period the diameters of clear inhabitation zones (Dizs) were measured in triplicates.

Negative control discs loaded with $20 \mathrm{ml}$ of the solvents were tested alongside the compounds while positive control discs containing specific affective concentrations of strptamycin, Methicillin and Erythromycin were tested in parallel. Results are recorded and expressed as mean of triplicate sets and DIZ were approximated to the nearest integrated value.

\section{Test Organisms}

For testing the antimicrobial activity of the compounds under study, four test bacterial species, namely Bacillus subtilis, Staphylococcus aureus ( $\mathrm{G}+\mathrm{ve})$, scherichia coli and Klebsiella pneumonia (Gram -ve) were used. All the above mentioned organisns were kindly gifted forms Clinical Microbiology Laboratory, Tripoli Medical Centre, Tripoli, Libya, while antimicrobial activity assessments were carried out by the technical Staff in Biolab, Chemistry Department, Faculty of Science, Tripoli University, and Tripoli, Libya.

\section{Results and Discussion}

\section{2-Aminothiophenol Derivatives}

The in vitro antibacterial activity of 2-aminothiophenol derivatives presented in (Table 2) using a limited spectrum of microbes belmg to Gram-positive and Gram-negative species. The results demonstrated that, among 5 tested individual 2 aminothiophenol, compounds (5b, c, d, and $11 \mathrm{~b}, \mathrm{c}$ ) displayed their highest activity against B. subtilis (D1Z 20-22 mm) at $500 \mu \mathrm{g} / \mathrm{disc}$. On the other hand compound No. (11c) showed mild anti G-positive potential while all tested compounds were completely inactive against both Gram-negative tested bacteria.

Table 2: In vitro antimicrobial activity of compounds (5b-d, and 11 b, c).

\begin{tabular}{|c|c|c|c|c|c|c|}
\hline \multicolumn{4}{|c|}{$\begin{array}{l}\text { Diameter of Inhibition Zone } \\
(\mathrm{mm})\end{array}$} & \multirow[t]{3}{*}{$\mu g \mid$ Disk } & \multirow[t]{3}{*}{ Solvent } & \multirow[t]{3}{*}{ Compd No. } \\
\hline \multicolumn{2}{|c|}{ Gram+ve } & \multicolumn{2}{|c|}{ Gram-ve } & & & \\
\hline B.sub & St.aur & E.coli & K.pne & & & \\
\hline -ve & -ve & 15 & 20 & 500 & Acetone & $5 b$ \\
\hline -ve & -ve & 15 & 21 & 500 & Acetone & $5 c$ \\
\hline -ve & $-v e$ & 15 & 20 & 500 & Acetone & $5 d$ \\
\hline -ve & $-v e$ & 18 & 22 & 500 & Acetone & $11 b$ \\
\hline -ve & $-v e$ & 15 & 17 & 500 & Acetone & $11 c$ \\
\hline -ve & $-v e$ & -ve & -ve & & Acetone & Control \\
\hline 9 & 9 & 20 & 20 & 10 & Disc & Streptomycin* \\
\hline
\end{tabular}

B.sub; Bacillus subtilis St.aur; Staphylococcus aureus; $\quad$ E.coli; Escherichia coli K.pne Klebsiella pneumonia, $\quad{ }^{*}$ Acts on DNA

Diaza bicyclo[7.2.1]dodeca derivatives 10b,c.

Table 3: In vitro antimicrobial activity of compounds (10 b, c):

B.sub Bacillus subtilis ;

\begin{tabular}{|c|c|c|c|c|c|c|}
\hline \multicolumn{4}{|c|}{$\begin{array}{l}\text { Diameter of Inhibition Zone } \\
(\mathrm{mm})\end{array}$} & \multirow[t]{3}{*}{$\mu g \mid$ Disk } & \multirow[t]{3}{*}{ Solvent } & \multirow[t]{3}{*}{ Compd No. } \\
\hline \multicolumn{2}{|c|}{ Gram+ve } & \multicolumn{2}{|c|}{ Gram-ve } & & & \\
\hline B.sub & St.aur & E.coli & K.pne & & & \\
\hline -ve & -ve & 11 & 12 & 500 & Acetone & $10 b$ \\
\hline -ve & -ve & 11 & 14 & 500 & Acetone & $10 c$ \\
\hline -ve & -ve & -ve & -ve & & Acetone & control \\
\hline 9 & 9 & 20 & 20 & 10 & Disc & Streptomycin* \\
\hline -ve & -ve & -ve & -ve & 5 & Disc & Methicillin** \\
\hline$-v e$ & -ve & -ve & -ve & 15 & Disc & Erythromycin*** \\
\hline
\end{tabular}

St.aur Staphylococcus aureus ; E.coli Escherichia coli K.pne Klebsiella pneumonia. 
${ }^{*}$ Acts on DNA ${ }^{* *}$ Acts on cell membrane $\quad{ }^{* * *}$ Acts on protein synthesis.

\section{Acknowledgments}

We are grateful to Libyan Petroleum Institute, International Center for Polymer Research, Central Laboratory, Cairo University, Egypt) for recording IR, ${ }^{1} \mathrm{H}$ NMR, ${ }^{13} \mathrm{C}$ NMR and Mass spectra and Prof. Abdel-Kader N. for biological activity Assessment.

\section{Experimental section}

All melting points were determined on a Koffler melting point apparatus and are uncorrected. ${ }^{1} \mathrm{H}$ NMR spectra were recorded on a Bruker avance $300 \mathrm{MHz}$ spectrometer using TMS as an internal reference (chemical shifts in $\square, \mathrm{ppm}$ ), ${ }^{13} \mathrm{C}$ NMR spectra were recorded on a Bruker avance $75 \mathrm{MHz}$ spectrometer using TMS as an internal reference (chemical shifts in $\square, \mathrm{ppm}$ ), IR in $\mathrm{KBr}$ were obtained on a Bruker FT-IR ISS 25 spectrophotometer (mmax in $\mathrm{cm}^{-1}$ ) and The Mass spectra were recorded on Shimadzu GCMS-QP 1000 EX (Japan) mass spectrometer at $70 \mathrm{eV}$.

(Z)-6-(2-aminophenythio)-3-(1,3-dithiolan--2-ylidene)-6-arylhex-5-ene-2,4-dione (5a-d).

\section{General Procedure}

A solution of $\alpha$-alkenoyl ketene- $(S, S)$-acetals 2 a-d $(1.0 \mathrm{mmol})$ in DMF $(5 \mathrm{ml})$ was added 2-aminothiophenole (3.0 mmol) in one portion at room temperature. The reaction mixture was stirred for $36 \mathrm{hrs}$ at room temperature and then poured into saturated sodium chloride aqueous $(25 \mathrm{ml})$. This was washed with water, filtered. The crude product was purified by petroleum ether to give the desired title compounds 5 a-d.

(Z)-6-(2-aminophenylthio)-3-(1,3-dithiolan-2-ylidene)-6-p-tolylhex-5-ene-2,4-dione 5a

( 83 \%), m.p. $101-102{ }^{\circ} \mathrm{C}$ [Found: C, 61.82; H, 5.01; N, 3.17; O, 7.50; S, 22.48, $\mathrm{C}_{22} \mathrm{H}_{21} \mathrm{NO}_{2} \mathrm{~S}_{3}$ (427.6) requires $\mathrm{C}, 61.79 ; \mathrm{H}$, 4.95; N, 3.28; O, 7.48; S, 22.50; IR ( $\left.\mathrm{v} \mathrm{cm}^{-1}\right)$ 3369, $3289\left(\mathrm{NH}_{2}\right), 3061\left(\mathrm{CH}_{\text {arom. }}\right), 2939\left(\mathrm{CH}_{\text {aliph. }}\right), 1695,1614(2 \mathrm{C}=\mathrm{O}) . \mathrm{cm}^{-1}$; ( $\left.{ }^{1} \mathrm{H}-\mathrm{NMR}, 300 \mathrm{MHz} \mathrm{CDC1}_{3}, \delta \mathrm{ppm}\right) 7.70-6.70$ (m, 8H, arom.), 6.90 (s, $\left.1 \mathrm{H}, \mathrm{CH}-\mathrm{S}\right), 4.43\left(\mathrm{~s}, 2 \mathrm{H}, \mathrm{NH}_{2}\right), 3.42\left(\mathrm{~m}, 4 \mathrm{H}, 2 \mathrm{CH}_{2}\right)$,

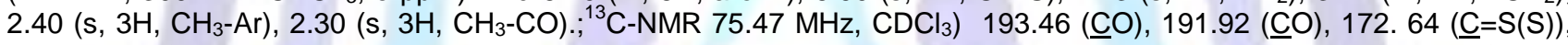

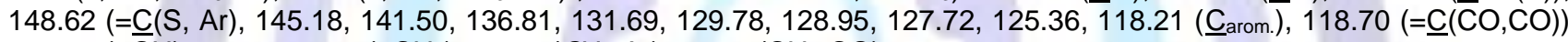
$115.23(=\underline{\mathrm{C}} \mathrm{H}), 37.81,36.79\left(2 \underline{\mathrm{C}} \mathrm{H}_{2}\right), 29.49\left(\mathrm{CH}_{3}-\mathrm{Ar}\right), 21.56\left(\underline{\mathrm{C}} \mathrm{H}_{3}-\mathrm{CO}\right)$.

(Z)-6-(2-aminophenylthio)-6-(4-chlorophenyl)-3-(1,3-dithiolan-2-ylidene)hex-5-ene-2,4-dione 5b

( 88\%), m.p. $104-108{ }^{\circ} \mathrm{C}$ [Found: C, 56.30; H, 4.05; Cl, 7.91; N, 3.13; O, 7.14; S, 21.47, $\mathrm{C}_{21} \mathrm{H}_{18} \mathrm{ClNO}_{2} \mathrm{~S}_{3}$ (448.02) requires C, 56.35; H, 3.98; Cl, 7.99; N, 3.11; O, 7.23; S, 21.40; IR $\left(\mathrm{v} \mathrm{cm}^{-1}\right) 3370,3280\left(\mathrm{NH}_{2}\right), 3006\left(\mathrm{CH}_{\text {arom. }}\right), 2930\left(\mathrm{CH}_{\text {aliph. }}\right), 1674$, 1620 (2C=O).). $\mathrm{cm}^{-1}$; ( $\left.{ }^{1} \mathrm{H}-\mathrm{NMR}, 300 \mathrm{MHz}, \mathrm{CDC} 1_{3}, \delta \mathrm{ppm}\right) 7.80-6.90(\mathrm{~m}, 8 \mathrm{H}$, arom.), $7.30(\mathrm{~s}, 1 \mathrm{H}, \mathrm{CH}), 4.50(\mathrm{~s}, 2 \mathrm{H}, \mathrm{NH})$,

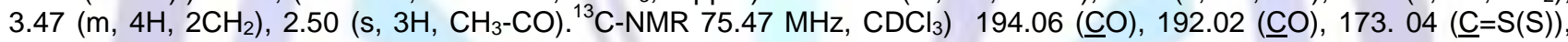

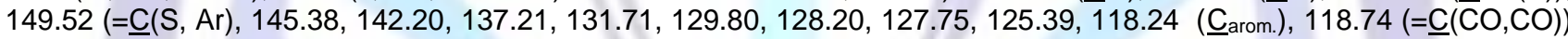
$115.23(=\underline{\underline{C}} \mathrm{H}), 38.31,38.12\left(2 \underline{\mathrm{C}} \mathrm{H}_{2}\right), 21.57\left(\underline{\mathrm{CH}}_{3}-\mathrm{CO}\right)$.

(Z)-6-(2-aminophenylthio)-3-(1,3-dithiolan-2-ylidene)-6-phenylhex-5-ene-2,4-dione 5c

( 66 \%), m.p. 87-90 ${ }^{\circ} \mathrm{C}$ [Found: C, 60.99; $\mathrm{H}, 4.63 ; \mathrm{N}, 3.39 ; \mathrm{O}, 7.74 ; \mathrm{S}, 23.26, \mathrm{C}_{21} \mathrm{H}_{19} \mathrm{NO}_{2} \mathrm{~S}_{3}$ (413.58) requires $\mathrm{C}, 60.89 ; \mathrm{H}$ 4.91 ; N, 3.32; O, 7.52; S, 22.48; IR $\left(\mathrm{v} \mathrm{cm}^{-1}\right) 3360,3298\left(\mathrm{NH}_{2}\right), 2983\left(\mathrm{CH}_{\text {arom. }}\right), 2930\left(\mathrm{CH}_{\text {aliph. }}\right), 1700,1665(2 \mathrm{C}=\mathrm{O}) . \quad \mathrm{cm}{ }^{-1}$ ( $\left.{ }^{1} \mathrm{H}-\mathrm{NMR}, 300 \mathrm{MHz} \mathrm{CDC1}_{3}, \delta \mathrm{ppm}\right) 7.74-6.76(\mathrm{~m}, 9 \mathrm{H}$, arom.), 6.92(s, $1 \mathrm{H}, \mathrm{CH}), 4.56\left(\mathrm{~s}, 2 \mathrm{H}, \mathrm{NH}_{2}\right), 3.54-3.34(\mathrm{~m}, 2 \mathrm{H}, 2 \mathrm{CH})$, 2.40 (s, $\left.\left.\mathrm{CH}_{3}-\mathrm{CO}\right) . .{ }^{13} \mathrm{C}-\mathrm{NMR} 75.47 \mathrm{MHz}, \mathrm{CDCl}_{3}\right) 193.16(\underline{\mathrm{CO}}), 191.82(\underline{\mathrm{CO}}), 173.64(\underline{\mathrm{C}}=\mathrm{S}(\mathrm{S})), 149.12(=\underline{\mathrm{C}}(\mathrm{S}, \mathrm{Ar}), 145.22$,

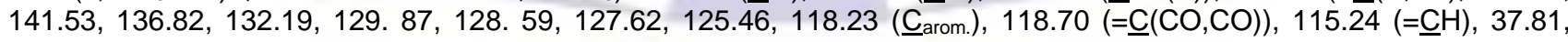
$37.79\left(2 \underline{\mathrm{C}} \mathrm{H}_{2}\right), 21.56\left(\underline{\mathrm{CH}}_{3}-\mathrm{CO}\right)$.

\section{(Z)-6-(2-aminophenylthio)-6-(4-(dimethylamino)phenyl)-3-(1,3-dithiolan-2-ylidene)hex-5-ene-2,4-dione 5d}

(87\%), m.p. 87-88 ${ }^{\circ} \mathrm{C}$ [Found: $\mathrm{C}, 60.49 ; \mathrm{H}, 5.30 ; \mathrm{N}, 6.13 ; \mathrm{O}, 7.01 ; \mathrm{S}, 21.07, \mathrm{C}_{23} \mathrm{H}_{24} \mathrm{~N}_{2} \mathrm{O}_{2} \mathrm{~S}_{3}$ (456.64) requires $\mathrm{C}, 60.54 ; \mathrm{H}$, 5.35; N, 6.10; O, 6.98; S, 21.00; IR ( $\left.\mathrm{v} \mathrm{cm}^{-1}\right)$ 3369, $3289\left(\mathrm{NH}_{2}\right), 3061\left(\mathrm{CH}_{\text {arom. }), 2939}\left(\mathrm{CH}_{\text {aliph. }}\right), 1695,1614(2 \mathrm{C}=\mathrm{O}) \mathrm{cm}^{-1}\right.$; ( $\left.{ }^{1} \mathrm{H}-\mathrm{NMR}, 300 \mathrm{MHz} \mathrm{CDC1}_{3}, \delta \mathrm{ppm}\right) 7.70-6.70\left(\mathrm{~m}, 8 \mathrm{H}\right.$, arom.), $6.90(\mathrm{~s}, 1 \mathrm{H}, \mathrm{CH}), 4.50\left(\mathrm{~s}, 2 \mathrm{H}, \mathrm{NH}_{2}\right), 3.40\left(\mathrm{~m}, 2 \mathrm{H}, 2 \mathrm{CH}_{2}\right)$, $2.40\left(\mathrm{~s}, 6 \mathrm{H}, \mathrm{N}\left(\mathrm{CH}_{3}\right)_{2}, 2.30\left(\mathrm{~s}, 3 \mathrm{H}, \mathrm{CH}_{3}-\mathrm{CO}\right) ;^{13} \mathrm{C}-\mathrm{NMR} 75.47 \mathrm{MHz}, \mathrm{CDCl}_{3}\right) 193.46(\underline{\mathrm{CO}}), 191.92$ (므), $172.64(\underline{\mathrm{C}}=\mathrm{S}(\mathrm{S}))$

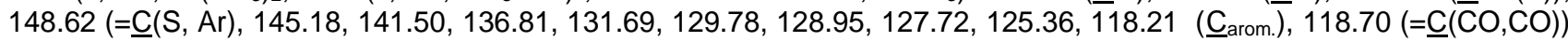
$\left.115.23(=\underline{\bar{C}} \mathrm{H}), 37.81,36.79\left(2 \underline{\mathrm{C}} \mathrm{H}_{2}\right), 29.49\left(\underline{\mathrm{CH}}_{3}\right) 2 \mathrm{~N}\right), 21.56\left(\underline{\mathrm{C}} \mathrm{H}_{3}-\mathrm{CO}\right)$.

\section{2-Acetyl-6-cyano-5-arylcyclohexspiro(1,2')-imidazolidine-3-one 6a-d}

\section{General Procedure}

To a solution of $\alpha$-alkenoyl ketene-S, $S$-acetal 2 a-d $(1 \mathrm{mmol})$ in DMSO $(5 \mathrm{ml})$ was added ammonium acetat $(2 \mathrm{mmol})$. The reaction mixture was heated to $100{ }^{\circ} \mathrm{C}$ in oil bath under stirring for $4 \mathrm{hrs}$. After cooling to room temperature, the 
mixtures were poured into saturated sodium chloride solution $(15 \mathrm{ml})$. The combined filtered, and. the crude products 6 a-d were recrystalized from ethanol.

2-Acetyl-6-cyano-5-(p-tolyl)-cyclohex spiro(1,2')-imidazolidine-3-one 6 a.

(75\%), m.p. 218-220 ${ }^{\circ} \mathrm{C}$ [Found: C, 69.43; $\mathrm{H}, 6.80 ; \mathrm{N}, 13.49 ; \mathrm{O}, 10.28 \mathrm{C}_{18} \mathrm{H}_{21} \mathrm{~N}_{3} \mathrm{O}_{2}(311.16)$ requires $\mathrm{C}, 69.27 ; \mathrm{H}, 6.79 ; \mathrm{N}$, 13.38; O, 10.38; IR ( $\left.\mathrm{v} \mathrm{cm}^{-1}\right) 3235(\mathrm{NH}), 3067\left(\mathrm{CH}_{\text {arom. }}\right), 2955,2912\left(\mathrm{CH}_{\text {aliph. }}\right), 2206(\mathrm{CN})$ 1627, $1573(2 \mathrm{C}=\mathrm{O}) \mathrm{cm}^{-1} ;\left({ }^{1} \mathrm{H}-\right.$ NMR, $\left.300 \mathrm{MHz} \mathrm{CDC1}_{3}, \delta \mathrm{ppm}\right) 7.20$ (s, 4H, arom.), $6.40(\mathrm{~s}, 1 \mathrm{H}, \mathrm{NH}), 4.60(\mathrm{~m}, 1 \mathrm{H}, \mathrm{CH}-\mathrm{Ar}), 3.07$ (s, 1H, CO-CH-CO), 2.60 (d, $\left.1 \mathrm{H}, \mathrm{CH}-\mathrm{CN}), 2.44\left(\mathrm{~s}, 3 \mathrm{H}, \mathrm{CH}_{3}-\mathrm{CO}\right), 2.35\left(\mathrm{~s}, 3 \mathrm{H}, \mathrm{CH}_{3}-\mathrm{Ar}\right), 1.70\left(\mathrm{~m}, 4 \mathrm{H}, 2 \mathrm{CH}_{2}\right) .{ }^{13} \mathrm{C}-\mathrm{NMR} 75.47 \mathrm{MHz}, \mathrm{CDCl} 3\right)$

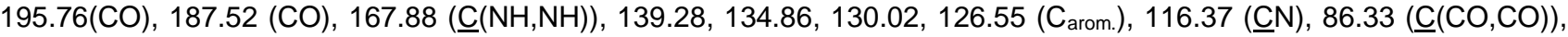

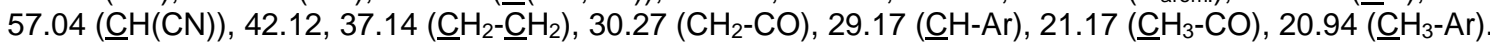

\section{2-Acetyl-6-cyano-5-(p-chlorophenyl)-cyclohexspiro(1,2')-imidazolidine-3-one $6 \mathrm{~b}$.}

(84\%) , m.p. $119-122{ }^{\circ} \mathrm{C}$ [Found: C, 61.54; H, 5.47; Cl, 10.69; N, 12.66; O, $9.64 \mathrm{C}_{17} \mathrm{H}_{18} \mathrm{Cl} \mathrm{N} \mathrm{O}_{2}$ (331.8) requires $\mathrm{C}, 61.60$ Cl, 10.71; H, 5.45; N, 12.68; O, 9.68; ; IR (v cm $\left.{ }^{-1}\right) 3206(\mathrm{NH}), 3067\left(\mathrm{CH}_{\text {arom. }}\right), 2913\left(\mathrm{CH}_{\text {aliph }}\right), 2207$ (CN), 1610, $1600(\mathrm{C}=\mathrm{O})$ $\mathrm{cm}^{-1}$; ( $\left.{ }^{1} \mathrm{H}-\mathrm{NMR}, 300 \mathrm{MHz} \mathrm{CDC1}_{3}, \delta \mathrm{ppm}\right) 7.30(\mathrm{~m}, 4 \mathrm{H}$, arom.), $6.02(\mathrm{~s}, 1 \mathrm{H}, \mathrm{NH}), 4.61(\mathrm{~m}, 1 \mathrm{H}, \mathrm{CH}-\mathrm{Ar}), 3.20(\mathrm{~s}, 1 \mathrm{H}, \mathrm{CO}-\mathrm{CH}-$ $\left.\mathrm{CO}), 2.61(\mathrm{~d}, 1 \mathrm{H}, \mathrm{CH}-\mathrm{CN}), 2.43\left(\mathrm{~s}, 3 \mathrm{H}, \mathrm{CH}_{3}-\mathrm{CO}\right), 1.72\left(\mathrm{~m}, 4 \mathrm{H}, 2 \mathrm{CH}_{2}\right) .{ }^{13} \mathrm{C}-\mathrm{NMR} 75.47 \mathrm{MHz}, \mathrm{CDCl}_{3}\right) 195.77(\mathrm{CO}), 187.55$ (CO), $167.87(\underline{\mathrm{C}}(\mathrm{NH}, \mathrm{NH})), 139.25,135.06,130.12,127.15,126.25\left(\mathrm{C}_{\text {arom. }}\right), 116.30(\underline{\mathrm{CN}}), 86.13(\underline{\mathrm{C}}(\mathrm{CO}, \mathrm{CO})), 57.06$ $(\underline{\mathrm{C}} \mathrm{H}(\mathrm{CN})), 42.15,37.20\left(\underline{\mathrm{C}} \mathrm{H}_{2}-\underline{\mathrm{C}} \mathrm{H}_{2}\right), 30.30\left(\mathrm{CH}_{2}-\mathrm{CO}\right), 29.20$ (ㅁH-Ar), $21.28\left(\underline{\mathrm{C}} \mathrm{H}_{3}-\mathrm{CO}\right)$.

2-Acetyl-6-cyano-5-phenyl-cyclohexspiro (1,2')-imidazolidine-3-one $6 c$.

( $58 \%$ ), m.p. $125-127{ }^{\circ} \mathrm{C}$ [FoundC, 68.67; $\mathrm{H}, 6.44 ; \mathrm{N}, 14.13 ; \mathrm{O}, 10.76 \mathrm{C}_{17} \mathrm{H}_{19} \mathrm{~N}_{3} \mathrm{O}_{2}$

(297.35) requires $\mathrm{C}, 68.73 ; \mathrm{H}$

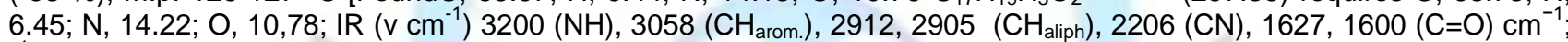
$\left({ }^{1} \mathrm{H}-\mathrm{NMR}, 300 \mathrm{MHz} \mathrm{CDC1}_{3}, \delta \mathrm{ppm}\right) 7.35(\mathrm{~s}, 5 \mathrm{H}$, arom.), $6.42(\mathrm{~s}, 1 \mathrm{H}, \mathrm{NH}), 4.63(\mathrm{~m}, 1 \mathrm{H}, \mathrm{CH}-\mathrm{Ar}), 3.17(\mathrm{~s}, 1 \mathrm{H}, \mathrm{CO}-\mathrm{CH}-\mathrm{CO})$, $\left.2.59(\mathrm{~d}, 1 \mathrm{H}, \mathrm{CH}-\mathrm{CN}), 2.43\left(\mathrm{~s}, 3 \mathrm{H}, \mathrm{CH}_{3}-\mathrm{CO}\right), 1.71\left(\mathrm{~m}, 4 \mathrm{H}, 2 \mathrm{CH}_{2}\right) ;{ }^{13} \mathrm{C}-\mathrm{NMR} 75.47 \mathrm{MHz}, \mathrm{CDCl}_{3}\right), 196.57$ (CO), $187.73(\mathrm{CO})$, $168.78(\underline{\mathrm{C}}(\mathrm{NH}, \mathrm{NH})), 139.25,134.06,131.02,127.15\left(\mathrm{C}_{\text {arom. }}\right), 116.50(\underline{\mathrm{CN}}), 87.13(\underline{\mathrm{C}}(\mathrm{CO}, \mathrm{CO})), 57.34(\underline{\mathrm{C}} \mathrm{H}(\mathrm{CN})), 43.12$, $38.14\left(\underline{\mathrm{C}} \mathrm{H}_{2}-\underline{\mathrm{CH}}_{2}\right), 30.20$ ( $\left.\mathrm{CH}_{2}-\mathrm{CO}\right), 29.23(\underline{\mathrm{C}} \mathrm{H}-\mathrm{Ar}), 21.28\left(\underline{\mathrm{CH}}_{3}-\mathrm{CO}\right)$.

\section{2-Acetyl-6-cyano-5-(p-(N,N-dimethylamino) phenyl)-cyclohexSpiro(1,2')-imidazolidine-3-one 6d).}

( 63 \%), m.p. $185-188^{\circ} \mathrm{C}$ [Found: $\mathrm{C}, 67.04 ; \mathrm{H}, 7.11 ; \mathrm{N}, 16.46 ; \mathrm{O}, 9.40, \mathrm{C}_{19} \mathrm{H}_{24} \mathrm{~N}_{4} \mathrm{O}$ (340.32) requires $\mathrm{C}, 67.11 ; \mathrm{H}, 7.18 ; \mathrm{N}$ 16.39; O, 9.48; IR ( $\left(\mathrm{v} \mathrm{cm}^{-1}\right) 3233(\mathrm{NH}), 3073\left(\mathrm{CH}_{\text {arom. }}\right), 2984,2816\left(\mathrm{CH}_{\text {aliph }}\right), 2208(\mathrm{CN}), 1650,1610(\mathrm{C}=\mathrm{O}) \mathrm{cm}^{-1} ;\left(^{1} \mathrm{H}-\mathrm{NMR}\right.$, $\left.300 \mathrm{MHz} \mathrm{CDC1}_{3}, \delta \mathrm{ppm}\right) 7.26(\mathrm{~s}, 4 \mathrm{H}$, arom.), $6.40(\mathrm{~s}, 1 \mathrm{H}, \mathrm{NH}), 4.61(\mathrm{~m}, 1 \mathrm{H}, \mathrm{CH}-\mathrm{Ar}), 3.07$ (s, $1 \mathrm{H}, \mathrm{CO}-\mathrm{CH}-\mathrm{CO}), 2.63(\mathrm{~d}, 1 \mathrm{H}$, $\left.\mathrm{CH}-\mathrm{CN}), 2.44\left(\mathrm{~s}, 6 \mathrm{H}, \mathrm{N}\left(\mathrm{CH}_{3}\right)_{2}\right), 2.35\left(\mathrm{~s}, 3 \mathrm{H}, \mathrm{CH}_{3}-\mathrm{CO}\right), 1.70\left(\mathrm{~m}, 4 \mathrm{H}, 2 \mathrm{CH}_{2}\right){ }^{13} \mathrm{C}-\mathrm{NMR} 75.47 \mathrm{MHz}, \mathrm{CDCl}_{3}\right) 195.77(\mathrm{CO})$, $187.53(\mathrm{CO}), 167.85(\underline{\mathrm{C}}(\mathrm{NH}, \mathrm{NH})), 139.25,135.06,130.12,127.15\left(\mathrm{C}_{\text {arom. }}\right), 116.30(\underline{\mathrm{CN}}), 86.13(\underline{\mathrm{C}}(\mathrm{CO}, \mathrm{CO})), 57.04$ $(\underline{\mathrm{C}} \mathrm{H}(\mathrm{CN})), 42.10,37.10\left(\underline{\mathrm{C}} \mathrm{H}_{2}-\underline{\mathrm{C}} \mathrm{H}_{2}\right), 30.30\left(\mathrm{CH}_{2}-\mathrm{CO}\right), 29.21(\underline{\mathrm{C}} \mathrm{H}-\mathrm{Ar}), 21.28\left(\underline{\mathrm{C}} \mathrm{H}_{3}-\mathrm{CO}\right), 21.22\left(\mathrm{~N}\left(\underline{\mathrm{C}} \mathrm{H}_{3}\right)_{3}\right)$.

\section{(3aZ,5Z,7Z)-3-Methylpyrazolo[4,3-d][1,2]-diazepine 8}

To a solution of 6-(4-(N,N-dimethylamino) phenyl)-3-(1,3-dithiolan-2-ylidine)-hex-5-ene-2,4-dione (2d) (1 mmol) in DMSO $(5 \mathrm{ml})$ was added hydrazine $(2 \mathrm{mmol})$. The reaction mixture was heated to $120^{\circ} \mathrm{C}$ in oil bath under stirring for 4 hrs. After cooling to room temperature, the mixture was poured into sodium chloride $(15 \mathrm{ml})$. The combined filtered, and. The crude product was purified by chloroform and petroleum ether $(2: 2)$, gave compound 8, ( $65.60 \%$, m.p. $253-255^{\circ} \mathrm{C}$ [Found: $\mathrm{C}, 57.53 ; \mathrm{H}, 4.14 ; \mathrm{N}, 38.34 ; \mathrm{C}_{7} \mathrm{H}_{6} \mathrm{~N}_{4}(148.16)$ requires $\mathrm{C}, 57.59 ; \mathrm{H}, 4.15 ; \mathrm{N}, 38.28 ; \mathrm{IR}\left(\mathrm{v} \mathrm{cm}{ }^{-1}\right) 3086\left(\mathrm{CH}_{\text {arom. }}\right)$, 2911, 2803 ( $\left.\mathrm{CH}_{\text {aliph. }}\right) . \mathrm{cm}^{-1}$; ( $\left.{ }^{1} \mathrm{H}-\mathrm{NMR}, 300 \mathrm{MHz} \mathrm{CDC1}_{3}, \delta \mathrm{ppm}\right) 8.60(\mathrm{~s}, 1 \mathrm{H}, \mathrm{CH}-\mathrm{N}), 7.80(\mathrm{~d}, 1 \mathrm{H}, \mathrm{CH}-\mathrm{N}), 6.70(\mathrm{~d}, 1 \mathrm{H}, \mathrm{CH}-$ $\left.\mathrm{C}=\mathrm{N}), 3.10\left(\mathrm{~s}, 3 \mathrm{H}, \mathrm{CH}_{3}\right) ; 7^{13} \mathrm{C}-\mathrm{NMR} 75.47 \mathrm{MHz}, \mathrm{CDCl}_{3}\right) 160.78\left(\mathrm{C}_{5}, \mathrm{C}=\mathrm{N}\right), 152.12\left(\mathrm{C}_{4}\right.$ pyrazole ring $), 129.19\left(\mathrm{C}_{6}\right.$, diazepine ring), $122.11\left(\mathrm{C}=\mathrm{N}\left(\mathrm{CH}_{3}\right), \mathrm{CH}\right), 111.72\left(\mathrm{C}_{5}\right.$, diazepine ring $), 76.60\left(\mathrm{C}_{3}\right.$, diazepine ring $), 40.20\left(\mathrm{CH}_{3}\right)$.

\section{9-Acetyl-5-phenyl-1-(phenylamino)-6-p-tolyl-2,3,6,7-tetrahydro-1H-imidazo[1,2-b][1,2]diazepin-8(5H)-one 9.}

To a solution of 3-(1,3-dithiolan-2-ylidine)-6-p-tolylhex-5-ene-2,4-dione $\mathbf{2 a}$ (1 mmol) in DMSO (5 ml), phenylhydrazine (2 $\mathrm{mmol}$ ) was added. The reaction mixture was heated to $120^{\circ} \mathrm{C}$ in oil bath under stirring for $2: 30 \mathrm{hrs}$. After cooling to room temperature, the mixture was poured into sodium chloride $(15 \mathrm{ml})$. The combined filtered, and. The crude product was purified by chloroform and petroleum ether, gave compound 9, $92.3 \%$ ) , m.p. 88-90 ${ }^{\circ} \mathrm{C}$ [Found $\mathrm{C}, 72.33 ; \mathrm{H}, 6.49 ; \mathrm{N}$, 14.54; O, 6.64, $\mathrm{C}_{29} \mathrm{H}_{31} \mathrm{~N}_{5} \mathrm{O}_{2}$ (481.59) requires C, 72.29; $\mathrm{H}, 6.45 ; \mathrm{N}, 14.58 ; \mathrm{O}, 6.68 ; \mathrm{IR}\left(\mathrm{v} \mathrm{cm}^{-1}\right) 3194(\mathrm{NH}), 2913\left(\mathrm{CH}_{\text {arom. }}\right)$, $2819\left(\mathrm{CH}_{\text {aliph. }}\right), 1625,1603(2 \mathrm{C}=\mathrm{O}) \mathrm{cm}^{-1}$; ( $\left.{ }^{1} \mathrm{H}-\mathrm{NMR}, 300 \mathrm{MHz} \mathrm{CDC1}_{3}, \delta \mathrm{ppm}\right) 7.70(\mathrm{~s}, 1 \mathrm{H}, \mathrm{NH}), 7.60-6.80(\mathrm{~m}, 14 \mathrm{H}$, arom. $)$, $3.35\left(\mathrm{~m}, 6 \mathrm{H}, 2 \mathrm{CH}_{2}, \mathrm{CH}_{2}\right), 3.10(\mathrm{~m}, 1 \mathrm{H}, \mathrm{CH}-\mathrm{Ar}), 2.39\left(\mathrm{~s}, 3 \mathrm{H}, \mathrm{CH}_{3}-\mathrm{Ar}\right), 2.30\left(\mathrm{~s}, 3 \mathrm{H}, \mathrm{CH}_{3}-\mathrm{CO}\right) . ;{ }^{3} \mathrm{C}-\mathrm{NMR} 75.47 \mathrm{MHz}^{\left.-\mathrm{CDCl}_{3}\right)}$ 195.96 (CO), 193.0 (CO), 172.67 ( $\underline{\mathrm{C}}(\mathrm{CO}, \mathrm{CO})), 146.34,145.15,144.12,143.25,141.49,138.36,131.68,129.54,129.43$, $129.21,128.81,126.74,125.86,123.14\left(\mathrm{C}_{\text {arom. }}\right), 37.80,37.50,37.13\left(\underline{3} \underline{\underline{C}} \mathrm{H}_{2}\right), 31.12(\underline{\mathrm{C}} \mathrm{H}-\mathrm{Ar}), 21.56\left(\underline{\mathrm{C}} \mathrm{H}_{3}-\mathrm{CO}\right)$.

\section{4,9-Diaryl-1,2,4,9-tetrahydroimidazo[3,2, 1ij][1,8]naphthyridine-6,7-diol 10a-c.}

\section{General Procedure}

To a solution of $\alpha, \alpha$-dialkenoyl ketene- $(S, S)$-acetals 3 a-c $(1.0 \mathrm{mmol})$ in DMF $(5 \mathrm{ml})$ ethylenediamine $(1.2 \mathrm{mmol})$ was added in one portion at room temperature. The reaction mixture was stirred for $48 \mathrm{hrs}$ at room temperature and then 
poured into saturated sodium chloride aqueous $(25 \mathrm{ml})$. This was washed with water, filtered. The crude product was purified by ethanol $+\mathrm{H}_{2} \mathrm{O}(2: 2)$, gave product 10a-c.

4,9-Di-p-tolyl-1,2,4,9-tetrahydroimidazo[3,2,1-ij][1,8] naphth-yridine-6,7-diol 10a.

( $85 \%$ ) , m.p. $192-196^{\circ} \mathrm{C}$ [Found: C, 77.39; H, 6.49; N, 7.52; O, 8.59, $\mathrm{C}_{24} \mathrm{H}_{24} \mathrm{~N}_{2} \mathrm{O}_{2}$ (372.46) requires C, 77.39; $\mathrm{H}, 6.53 ; \mathrm{N}$, 7.58; O, 8.58; IR ( $\left.\mathrm{v} \mathrm{cm}^{-1}\right) 3286(\mathrm{OH}), 3020\left(\mathrm{CH}_{\text {arom. }}\right), 2918\left(\mathrm{CH}_{\text {aliph. }}\right) \mathrm{cm}^{-1}$; ( $\left.{ }^{1} \mathrm{H}-\mathrm{NMR}, 300 \mathrm{MHz} \mathrm{CDC1}_{3}, \delta \mathrm{ppm}\right) 9.50(\mathrm{~s}, 1 \mathrm{H}$, $\mathrm{OH}$ ), 7.60-7.09 (m, 8H, arom.), $6.90(\mathrm{~d}, 2 \mathrm{H}, 2 \mathrm{CH}=\mathrm{C}-\mathrm{OH}), 3.70$ (d, 2CH, 2CH-Ar), 2.40 (s, 4H, 2CH $), 2.30(\mathrm{~s}, 6 \mathrm{H}$, $\left.\left.2 \mathrm{CH}_{3}\right) ;{ }^{13} \mathrm{C}-\mathrm{NMR} 75.47 \mathrm{MHz}, \mathrm{CDCl}_{3}\right) 188.68(\underline{\mathrm{C}}(\mathrm{OH})), 166.71(\underline{\mathrm{C}}(\mathrm{N}, \mathrm{N})), 138.64$ (C-Ar), 139.74, 132.87, 129.88, 128.46, ( $\left.\mathrm{C}_{\text {arom. }}\right), 101.74(\underline{\mathrm{C}}(\mathrm{C}-\mathrm{OH}, \mathrm{C}-\mathrm{OH})), 77.90\left(\mathrm{CH}_{\text {Pyridine, }} \mathrm{CH}\right), 46.64\left(2 \underline{\mathrm{C}} \mathrm{H}_{2}\right), 21.15\left(\underline{\mathrm{C}} \mathrm{H}_{3}\right)$.

4,9-Bis(4-chlorophenyl)-1,2,4,9-tetrahydroimidazo[3,2,1-ij]- [1,8]naphthyridine-6,7-diol 10 b.

( $83 \%$ ), m.p. $134-138^{\circ} \mathrm{C}$ [Found: $\mathrm{C}, 63.93 ; \mathrm{H}, 4.39 ; \mathrm{Cl}, 17.16 ; \mathrm{N}, 6.78 ; \mathrm{O}, 7.74, \mathrm{C}_{22} \mathrm{H}_{18} \mathrm{Cl}_{2} \mathrm{~N}_{2} \mathrm{O}_{2}$ (413.3) requires $\mathrm{C}, 64.11 ;$ $\mathrm{H}, 4.35 ; \mathrm{N}, 6.68 ; \mathrm{O}, 7.78$; IR $\left(\mathrm{v} \mathrm{cm}^{-1}\right) 3286(\mathrm{OH}), 3020\left(\mathrm{CH}_{\text {arom. }}\right), 2918\left(\mathrm{CH}_{\text {aliph. }}\right) \mathrm{cm}^{-1} ;\left({ }^{1} \mathrm{H}-\mathrm{NMR}, 300 \mathrm{MHz} \mathrm{CDC} 1_{3}, \delta \mathrm{ppm}\right)$ $9.60(\mathrm{~s}, 1 \mathrm{H}, \mathrm{OH}), 7.60-7.09\left(\mathrm{~m}, 8 \mathrm{H}\right.$, arom.), $6.80(\mathrm{~d}, 2 \mathrm{H}, 2 \mathrm{CH}=\mathrm{C}-\mathrm{OH}), 3.80(\mathrm{~d}, 2 \mathrm{CH}, 2 \mathrm{CH}-\mathrm{Ar}), 2.40(\mathrm{~s}, 4 \mathrm{H}, 2 \mathrm{CH}) ;{ }^{13} \mathrm{C}-\mathrm{NMR}$ $\left.75.47 \mathrm{MHz} \mathrm{CDCl}_{3}\right) \quad 188.78(\underline{\mathrm{C}}(\mathrm{OH})), 167.51(\underline{\mathrm{C}}(\mathrm{N}, \mathrm{N})), 139.14(\underline{\mathrm{C}}-\mathrm{Ar}), 138.94,133.17,129.85,128.46\left(\mathrm{C}_{\text {arom. }}\right), 102.04$ $(\underline{\mathrm{C}}(\mathrm{C}-\mathrm{OH}, \mathrm{C}-\mathrm{OH})),), 77.86\left(\mathrm{CH}_{\text {Pyridine }}, \mathrm{CH}\right), 47.64\left(2 \underline{\mathrm{C}} \mathrm{H}_{2}\right)$.

\section{4,9-Diphenyl-1,2,4,9-tetrahydroimidazo[3,2,1-ij][1,8] naphth-yridine-6,7-diol 10c.}

(72 \%), m.p. $120-122{ }^{\circ} \mathrm{C}$ [Found: $\mathrm{C}, 76.72 ; \mathrm{H}, 5.85 ; \mathrm{N}, 8.13 ; \mathrm{O}, 9.29, \mathrm{C}_{22} \mathrm{H}_{20} \mathrm{~N}_{2} \mathrm{O}_{2}$ (344.41) requires $\mathrm{C}, 76.79 ; \mathrm{H}, 5.90 ; \mathrm{N}$, 8.18; O, 9.28; IR ( $\left.\mathrm{v} \mathrm{cm}^{-1}\right) 3282(\mathrm{OH}), 3020\left(\mathrm{CH}\right.$ arom.), $2918\left(\mathrm{CH}_{\text {aliph. }}\right) \mathrm{cm}^{-1}$; ( $\left.{ }^{1} \mathrm{H}-\mathrm{NMR}, 300 \mathrm{MHz} \mathrm{CDC1} 1_{3}, \delta \mathrm{ppm}\right) 9.60(\mathrm{~s}$, $1 \mathrm{H}, \mathrm{OH}), 7.70-7.23(\mathrm{~m}, 9 \mathrm{H}$, arom. $), 6.89(\mathrm{~d}, 2 \mathrm{H}, 2 \mathrm{CH}=\mathrm{C}-\mathrm{OH}), 3.70(\mathrm{~d}, 2 \mathrm{CH}, 2 \mathrm{CH}-\mathrm{Ar}), 2.50\left(\mathrm{~s}, 4 \mathrm{H}, 2 \mathrm{CH}_{2}\right) ;{ }^{13} \mathrm{C}-\mathrm{NMR}^{75.47}$ $\left.\mathrm{MHz}_{\mathrm{CDCl}}\right) 188.77(\underline{\mathrm{C}}(\mathrm{OH})), 166.73(\underline{\mathrm{C}}(\mathrm{N}, \mathrm{N})), 139.14(\underline{\mathrm{C}}-\mathrm{Ar}), 138.94,133.17,129.45,129.85,128.46\left(\mathrm{C}_{\text {arom. }}\right), 100.16$ $(\underline{\mathrm{C}}(\mathrm{C}-\mathrm{OH}, \mathrm{C}-\mathrm{OH})),), 77.93\left(\mathrm{CH}_{\text {Pyridine }}, \mathrm{CH}\right), 47.54\left(2 \underline{\mathrm{CH}}_{2}\right)$.

\section{1,7-Bis(2-aminophenylthio)-4-(1,3-dithiolane-2-ylidine)-1,7-diary1hepta-1,6-diene-3,5-dione 11a-c \\ General Procedure}

To a solution of $\alpha, \alpha$-dialkenoyl ketene- $(S, S)$-acetal 3a-c $(1.0 \mathrm{mmol})$ in DMF $(5 \mathrm{ml})$ 2-aminothiophenol $(3.0 \mathrm{mmol})$ was added in one portion at room temperature. The reaction mixture was stirred for $36 \mathrm{hrs}$ at room temperature and then poured into saturated sodium chloride aqueous $(25 \mathrm{ml})$. This was washed with water, filtered. The crude products were purified by ethanol $+\mathrm{H}_{2} \mathrm{O}(1: 1)$, gave products $11 \mathrm{a}-\mathrm{c}$.

\section{1,7-Bis(2-aminophenylthio)-4-(1,3-dithiolane-2-ylidine)-1,7-di-p-toly1 hepta-1,6-diene-3,5-dione 11 .}

( 70 \%), m.p. $100-104{ }^{\circ} \mathrm{C}$ [Found: C, 66.22; H, 4.94; N, 4.29; O, 4.90; S, 19.64, $\mathrm{C}_{36} \mathrm{H}_{32} \mathrm{~N}_{2} \mathrm{O}_{2} \mathrm{~S}_{4}$ (652.91) requires C, 66.30; $\mathrm{H}, 4.95$; N, 4.28; O, 4.88; IR ( $\left.\mathrm{v} \mathrm{cm}^{-1}\right) 3372,3292\left(\mathrm{NH}_{2}\right), 3061\left(\mathrm{CH}_{\text {arom. }}\right), 2919\left(\mathrm{CH}_{\text {aliph. }}\right), 1621(\mathrm{CO}) \cdot \mathrm{cm}^{-1} ;\left(^{1} \mathrm{H}-\mathrm{NMR}, 300\right.$ $\left.\mathrm{MHz} \mathrm{CDC1}_{3}, \delta \mathrm{ppm}\right) 7.50-6.50(\mathrm{~m}, 18 \mathrm{H}, 16 \mathrm{H}$ arom $+2 \mathrm{H}$ of $2 \mathrm{CH}), 4.10\left(\mathrm{br}, 4 \mathrm{H}, 2 \mathrm{NH}_{2}\right), 3.40\left(\mathrm{~s}, 4 \mathrm{H}, 2 \mathrm{CH}_{2}\right), 2.53(\mathrm{~s}, 6 \mathrm{H}$, $\left.\left.2 \mathrm{CH}_{3}\right) . ;{ }^{13} \mathrm{C}-\mathrm{NMR} 75.47 \mathrm{MHz}, \mathrm{CDCl}_{3}\right) 187.86(\underline{\mathrm{CO}}), 148.64(\underline{\mathrm{C}}=\mathrm{S}(\mathrm{S})), 148.59$ (= $\underline{\mathrm{C}}(\mathrm{S}, \mathrm{Ar}), 143.83,136.74,134.61,131.62$,

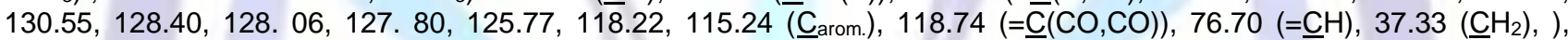
$22.17\left(\mathrm{CH}_{3}-\mathrm{Ar}\right)$.

\section{1,7-Bis(2-aminophenylthio)-1,7-bis(4-chlorophenyl)-4-(1,3-dithiolane-2-ylidine)-hepta-1,6-diene-3,5-dione $11 \mathrm{~b}$.}

( $64 \%$ ), m.p. $89-90{ }^{\circ} \mathrm{C}$ [Found: C, 58.86; $\mathrm{H}, 3.78 ; \mathrm{Cl}, 10.22 ; \mathrm{N}, 4.04 ; \mathrm{O}, 4.61 ; \mathrm{S}, 18.49, \mathrm{C}_{34} \mathrm{H}_{26} \mathrm{Cl}_{2} \mathrm{~N}_{2} \mathrm{O}_{2} \mathrm{~S}_{4}$ (693.75) requires C, 58.79; H, 3.75; Cl, 10.11; N , 4.08; O, 4.63; S, 18.50; IR $\left(\mathrm{v} \mathrm{cm}^{-1}\right) 3372,3294\left(\mathrm{NH}_{2}\right), 3063,3022\left(\mathrm{CH}_{\text {arom. }}\right), 2925\left(\mathrm{CH}_{\text {aliph }}\right)$, 1623 (CO) $\mathrm{cm}^{-1}$; ( $\left.{ }^{1} \mathrm{H}-\mathrm{NMR}, 300 \mathrm{MHz} \mathrm{CDC1}_{3}, \delta \mathrm{ppm}\right) 7.70-6.50(\mathrm{~m}, 18 \mathrm{H}, 16 \mathrm{H}$ arom $+2 \mathrm{H}$ of $2 \mathrm{CH}), 3.80\left(\mathrm{br}, 4 \mathrm{H}, 2 \mathrm{NH}_{2}\right), 3.35$

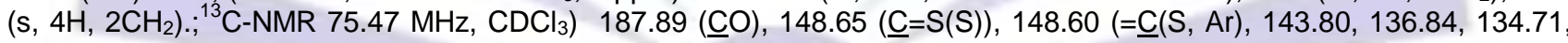

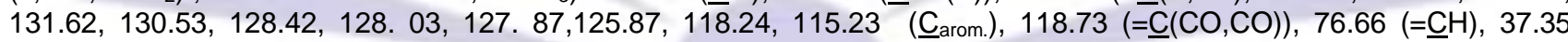
$\left(\underline{\mathrm{C}} \mathrm{H}_{2}\right)$.

\section{1,7-Bis(2-aminophenylthio)-4-(1,3-dithiolane-2-ylidine)-1,7-diphenylhepta-1,6-dione-3,5-diene 11c.}

(81 \%), m.p. $109-11{ }^{\circ} \mathrm{C}$ [Found: C, 65.35; H, 4.52; N, 4.48; O, 5.12; S, 20.53, C34H28N2O2S4 (624.86) requires C,

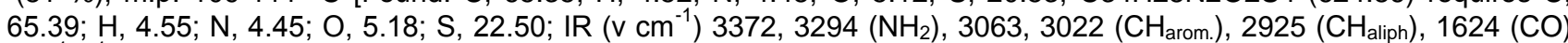
$\mathrm{cm}^{-1}$; ( $\left.{ }^{1} \mathrm{H}-\mathrm{NMR}, 300 \mathrm{MHz} \mathrm{CDC1}_{3}, \delta \mathrm{ppm}\right) 7.80-6.50(\mathrm{~m}, 2 \mathrm{H}, 18 \mathrm{H}$ arom $+2 \mathrm{H}$ of $2 \mathrm{CH}), 4.40\left(\mathrm{br}, 4 \mathrm{H}, 2 \mathrm{NH}_{2}\right), 3.40(\mathrm{~s}, 4 \mathrm{H}$, $\left.\left.2 \mathrm{CH}_{2}\right) ;{ }^{13} \mathrm{C}-\mathrm{NMR} 75.47 \mathrm{MHz}, \mathrm{CDCl}_{3}\right) 187.83(\underline{\mathrm{CO}}), 148.62(\underline{\mathrm{C}}=\mathrm{S}(\mathrm{S})), 148.60$ (= $\mathrm{C}(\mathrm{S}, \mathrm{Ar}), 143.81,136.84,134.71,131.62$,

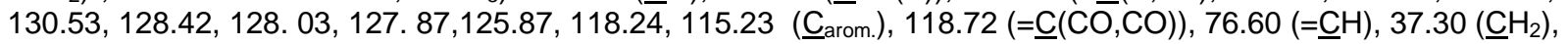

\section{3-Methyl-6-p-tolyl-6H-pyrazolo[3,4-b]pyridin-4-ol 13.}

A solution of 3,3-(methylthiophenylamino)methylene)-6-p-tolylhexa-5-ene-2,4-dione (12) (1 $\mathrm{mmol})$ in DMSO (5 ml) hydrazine $(1 \mathrm{mmol})$ was added. The reaction mixture was heated to $120^{\circ} \mathrm{C}$ in oil bath under stirring for $2 \mathrm{hrs}$. After cooling to room temperature, the mixture was poured into sodium chloride $(15 \mathrm{ml})$. The combined filtered, and the crude product was purified by $\mathrm{CHCl}_{3}+$ pet. Ether (1:3), gave compound 13, $(60.38 \%)$, m.p. $242-243^{\mathrm{d}}{ }^{\circ} \mathrm{C}$ [Found: $\mathrm{C}, 70.28 ; \mathrm{H}, 5.48 ; \mathrm{N}$, 17.56; O, 6.69, $\mathrm{C}_{14} \mathrm{H}_{13} \mathrm{~N}_{3} \mathrm{O}(239.27)$ requires C, 70.29; $\mathrm{H}, 5.45 ; \mathrm{N}, 17.58 ; \mathrm{O}, 6.68 ; \mathrm{IR}\left(\mathrm{v} \mathrm{cm}^{-1}\right) 3322(\mathrm{OH}), 3095(\mathrm{CH}$ arom.), $2932\left(\mathrm{CH}_{\text {aliph }}\right) \mathrm{cm}^{-1}$; ( $\left.{ }^{1} \mathrm{H}-\mathrm{NMR}, 300 \mathrm{MHz} \mathrm{CDC1}_{3}, \delta \mathrm{ppm}\right) 9.40(\mathrm{~s}, 1 \mathrm{H}, \mathrm{OH}), 7.50-7.30(\mathrm{~m}, 4 \mathrm{H}$, arom. $), 6.90(\mathrm{~d}, 1 \mathrm{H}, \mathrm{CH}=\mathrm{C})$, $\left.2.61(\mathrm{~s}, 1 \mathrm{H}, \mathrm{CH}) 2.40\left(\mathrm{~s}, 3 \mathrm{H}, \mathrm{CH}_{3}-\mathrm{Ar}\right), 2.53\left(\mathrm{~s}, 3 \mathrm{H}, \mathrm{CH}_{3} \mathrm{CO}\right) ;{ }^{13} \mathrm{C}-\mathrm{NMR} 75.47 \mathrm{MHz}, \mathrm{CDCl}_{3}\right) \quad 193.78(\mathrm{C}-\mathrm{OH}), \quad 154.00$ 


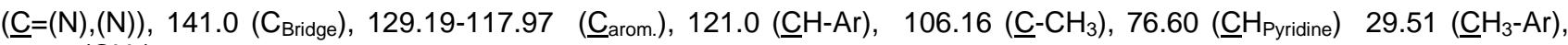
$14.11\left(\underline{\mathrm{C}} \mathrm{H}_{3}\right)$.

\section{REFERENCES}

[1] Kelber C. Ber. Dtsch. Chem. Ges.; 1910, 43, 1252

[2] a).Thuillier A., Vialle J.; Bull. Soc. Chim. Fr. 1962, 2187-2193; b)

Thuillier A., Vialle J.; Bull. Soc. Chim. Fr.; 1962, $2194-2198$.

[3] a). Larsson F.; Lawesson S.; Tetrahedron.; 1972,28, 5341-5347; b). Corey E. J.R. Chen H. K.; Tetrahedron Lett.; 1973, 14, 3817-3820; c). Dieter R. K.; J. Org.Chem.; 1981, 46, 5031- 5033.

[4] a). Dalgaard L., Kolind-Andersen H., Lawesson S.; Tetrahedron.; 1973, 29,2077-2085;b). Dalgaard L.,Jensen L., Lawesson S.O.; Tetrahedron.; 1974, 30, 93 -104.

[5] a). Breslow R., Rideout D. C.; J. Am. Chem. Soc. ; 1980, 102,7816-7817; b). Breslow R.; Acc. Chem. Res.;1991,24, $159-164$.

[6] a). Aqueous-Phase Organometallic Catalysis. Conceptsand Applications ; Eds.: (Cornils, B. Herrmann W. A.),WileyVCH, Weinheim ,1998;b). Organic Synthesis in Water; (Ed.:P. A. Grieco), Blackie Academic and Professional:London, 1998.;c). Li C., Chan T., Organic Reactionsin Aqueous Media ; John Wiley \& Sons, NewYork,1997;d).Kobayashi S., Manabe K.; In Stimulating Concepts in Chemistry.; (Eds.: Shibasaki M., Stoddart J., Vogtle F.).; Wiley-VCH, Weinheim.; 2000.

[7] a). Li C.; Chem. Rev.; 1993, 93, 2023-2035.; b).Genet J.; Savignac M.; J. Organomet. Chem.; 1999, 576, 305317;c). Fringuelli F., Piermatti O., Pizzo F., Vaccaro L.; Eur. J.Org. Chem.; 2001, 439 -452; d). Kobayashi S., Manabe,K.; Acc. Chem. Res.; 2002, 35, 209 - 217.

[8] a). Kobayashi S.; Wakabayashi T.; Tetrahedron Lett.; 1998,39, 5389 -5392.; b). Manabe K., Mori Y., Kobayashi S.; Synlett.; 1999, 1401-1402.; c). Manabe K., Kobayashi S.; Org. Lett. ;1999, 1, 1965-1967; d). Otto S., Engberts J., Kwak J.; J. Am. Chem. Soc.; 1998, 120, 9517 -9525.; e). Rispens T., Engberts J.; Org. Lett. 2001, 3, 941-943.; f). Manabe K., Sun X., Kobayashi S.; J.Am. Chem. Soc.; 2001, 123, $10101-10102$.

[9] El_Shafei A., El-Saghier A. M, Ahmed E.; Synthesis;1994, 2,152-154.

[10] Ouyang Y., Dong D., Pan W. , Zhang J. and Liu Q., J. Tetrahedron.; 2006, 62,10111-10116.

[11] Huang Z., Liu Z.; Synthesis.; 1978, 357

[12] Gompper R ., Toep ft.W.; cheme .Ber.; 1962, 95, 2861.

[13] Hiria K., Matsuda H., Kishda Y.; chem. Pharm. Bull.; 1971, 20,97.

[14] Mansour N., Rudor W., Augustian M.; Chem.; 1981, 21, 69.

[15] Rajappa S., Advani B.; Proc.Indian Acad.Sci.(chem.Sci.).; 982, 91, 463.

[16] Huang Z., Shi X.; Synthesis.; 1990,162.

[17] a). Dieter R. K.; Tetrahedron.; 1986, 42, 3029-3096.;b). Tominaga Y.; J. Heterocycl. Chem.; 1989, $26,1167-$ 1204.;c). Junjappa H., Ila H., Asokan C. V.;Tetrahedron.; 1990, 46, 5423 - 5506;d). Kolb M.; Synthesis.; 1990, 171190.; e).Junjappa H., Ila .H.; Phosphorus, Sulfur Silicon .;1994, 35, 95 - 96; f)., Junjappa H., Ila. H., Mohanta P.; in: Progress in Heterocyclic Chemistry.;(Eds.:Gribble G., Gilchrist L. ); Pergamon Press, Oxford.; 2001, (13), Ch.1, 1 -24 .

[18] a). Singh G., Purkayastha M., lla H., Junjappa H.; J. Chem. Soc. $\quad$ Perkin Trans .1; 1985, 1289-1294.; b). Kocienski P., Pontiroli J., Liu Q.; J.Chem .Soc. Perkin Trans. 1; 2001, 2356-2366; c). Asokan C., Junjappa V., lla H.; Synthesis. ; 1987, $284-285$

[19] a). Apparao S., Datta A. ,Ila H.; Synthesis.; 1985, 169 - 171; b). Datta A., Ila H., Junjappa H.; Tetrahedron Lett.; 1988, 29, 497-500.

[20] a). Balu M., Ila H., Junjappa H.; Tetrahedron Lett.; 1987, 28, 3023 - 3026.; b). Dieter R., Fishpaugh J.; J. Org. Chem.; 1988, 53, 2031- 2046.

[21] a). Dieter R., Silks L , Fishpaugh J., Kastner M .; J. Am. Chem. Soc. 1985, 107, 4679 - 4692.; b). Dieter R., Fishpaugh J., Silks L.; Tetrahedron Lett.1982, 23, 3751-3754.

[22] El-Saghier A. M. M., Alwedi E. F. and Fawzy N. M., J. Heterocyclic Chem., 2012, 49, 664.

[23] Khodairy A. and El-Saghier A. M., Acta Chim. Solv., 2011, 58, 360-366.

[24] Mohamed M. A. A., Fawzy N. M., and El-Saghier A. M., Chemistry J., 2012, 2(2), 101-105.

[25] Amed E. A., Mohamed M. A. A. and El-Saghier A. M., J. Am. Sci., 2012, 8(8), 815-818 
[26] Farhat M. F., Makhlouf M. A., El-Saghier A. M., Mezoughi A. B. A., Awhida S. M. and El-Mehdi A. M., Arb. J. Chem., 2011, 4, 307-3011.

[27] For Review: El-Saghier A. M., Abdel-Ghany H, Mohamed A. A. M. and Younes S. H., Trend Org. Chem., 2011, 15, 124.

[28] Bauer A. W., Kirby M. M. , Sherris J. C. and Truck M., Antibiotic Susceptibility testing by a Standardized single disk method., Am. J. Clin. Pathol, 1966, 45, 493-6. 